Alberta Thy 11-04

UVIC-TH-04/07

\title{
Electric Dipole Moments of Leptons in the Presence of Majorana Neutrinos
}

\author{
John Paul Archambault ${ }^{1}$, Andrzej Czarnecki ${ }^{1}$ and Maxim Pospelov ${ }^{2}$ \\ ${ }^{1}$ Department of Physics, University of Alberta, \\ Edmonton, AB, T6G 2J1 Canada \\ ${ }^{2}$ Department of Physics and Astronomy, University of Victoria, \\ Victoria, BC, V8P $1 A 1$ Canada
}

\begin{abstract}
We calculate the two-loop diagrams that give a non-zero contribution to the electric dipole moment $d_{l}$ of a charged lepton $l$ due to possible Majorana masses of neutrinos. Using the example with one generation of the Standard Model leptons and two heavy right-handed neutrinos, we demonstrate that the non-vanishing result for $d_{l}$ first appears in order $O\left(m_{l} m_{\nu}^{2} G_{F}^{2}\right)$, where $m_{\nu}$ is the mass of the light neutrino and the see-saw type relation is imposed. This effect is beyond the reach of presently planned experiments.
\end{abstract}




\section{Introduction}

Recent discovery of $\mathrm{CP}$ violation in the neutral $B$-meson system $[1,2]$ is in perfect accord with the $\mathrm{CP}$ violation observed in $K$ mesons [3]. Both results are explained within the minimal model of CP violation, known as the Kobayashi-Maskawa model [4]. It links CP-violation in $K$ and $B$ mesons to a single CP-violating invariant of the KobayashiMaskawa matrix in the quark sector, $\operatorname{Im}\left(V_{t b} V_{t d}^{*} V_{c d} V_{c b}^{*}\right) \simeq 3 \times 10^{-5}$. This combination, as well as $\theta_{\mathrm{QCD}}$, are the only sources of $\mathrm{CP}$ violation in the Standard Model.

An independent piece of experimental information about $\mathrm{CP}$ violation comes from the searches of the electric dipole moments (EDMs) of neutrons [5] and heavy atoms [6, 7]. It is well known that the Kobayashi-Maskawa model predicts extremely small EDMs. Indeed, the necessity of four electroweak vertices requires from any diagram capable of inducing an EDM of a quark to have at least two loops. Moreover, it turns out that all EDMs or color EDMs of quarks vanish exactly at the two-loop level [8], and only three-loop diagrams survive $[9,10]$, producing a tiny number of order $10^{-34} \mathrm{e} \mathrm{cm}$.

The only relevant operator that is not zero at the two-loop order is the Weinberg operator [11], but its numerical value turns out to be also extremely small. Possible enhancement comes from the large distance effects, that could lead to a KM-generated EDM of the neutron of order $10^{-32} \mathrm{e} \mathrm{cm}[12]$, which is still six to seven orders of magnitude smaller than the current experimental limit. The KM phase in the quark sector induces the EDM of a lepton via a diagram with a closed quark loop, but a non-vanishing result appears first at a four-loop level [13] and therefore is even more suppressed.

The suppressed values of EDMs from the KM model together with enormous accuracy of EDM experiments produce stringent constraints on possible flavour-diagonal sources of CP violation, notably on CP-odd combinations of the soft-breaking parameters in the supersymmetric extensions of the Standard Model (SM).

On the other hand, the KM phase cannot be the only source of $\mathrm{CP}$ violation in nature. Dynamical generation of the baryon asymmetry of the Universe (BAU) requires presence of an additional source(s) of $\mathrm{CP}$ violation in nature. One of the most appealing scenarios for BAU is leptogenesis [14] where a non-zero lepton number is generated from the out-ofequilibrium decay of heavy right-handed Majorana neutrinos with $\mathrm{CP}$ violation coming from the Yukawa sector of the theory. Subsequent sphaleron transitions [15] transform half of the initial lepton asymmetry into BAU.

The attractiveness of leptogenesis is in its simplicity, relative freedom from the low-energy constraints, and in recent experimental results that indicate large mixing angles in the lepton sector [16].

The complex phases in the lepton mixing suggested by leptogenesis at some level will induce $d_{e}$, the electron EDM, as well as EDMs of other charged leptons. Various calculations of $d_{e}$ and $d_{\mu}$ were performed in the supersymmetric case under certain assumption about the soft-breaking parameters [19], while a non-supersymmetric case remains poorly explored.

We note that manifestations of Majorana phases in CP-violating phenomena has been explored in [20]. The EDM is an additional observable arising due to those phases. Compared with the KM model, where three generations and at least four loops are necessary 
to generate a lepton EDM, the addition of Majorana phases enhances the effect. However, as we will see, it remains very small.

In this paper, we present a systematic analysis of the two-loop diagrams that lead to the EDMs of charged leptons through interactions with two additional heavy neutrinos, in a one-generation model suggested by a minimal leptogenesis scenario. These diagrams can be divided into two major classes. The first class where the lepton number is conserved along the fermion line vanishes identically, as the same arguments that lead to $d_{q}=0$ at two loops [8] apply (see Fig. 1).

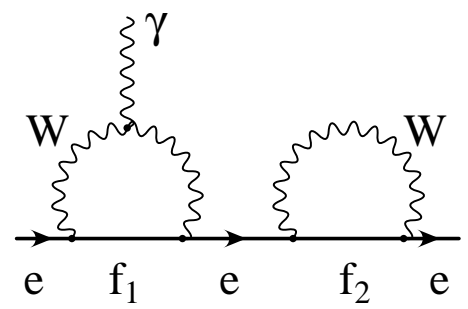

(a)

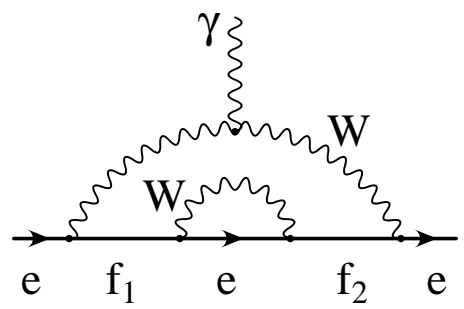

(b)

Figure 1: Examples of two-loop diagrams that give zero electric dipole moment of the electron. The direction of the arrows remains the same on all fermion propagators and the external photon can be attached to any charged line.

The second class of diagrams (Fig. 2) has no analogues in the quark sector: due to the existence of the Majorana component of the neutrino mass matrix, a combination of $\Delta L=1$ and $\Delta L=-1$ transitions is possible. The existence of this additional class of diagrams for leptons was pointed out in Ref. [17]. It leads to a non-zero electron EDM $\left(d_{e}\right)$ even at the two-loop level, but up to now there has not been any serious attempt to calculate the size of $d_{e}$ induced by this class of diagrams. (An estimate of $d_{e}$ due to CP violation in the sector of heavy Majorana neutrino was presented in Ref. [18]. However, this result is not satisfactory, as it suggests that $d_{e}$ does not vanish in the limit of infinitely heavy right-handed neutrinos.)

The purpose of this work is to give a detailed calculation of $d_{e}$ due to the CP violation in the lepton sector in the presence of Majorana masses for neutrinos and obtain a prediction for the electron EDM in the see-saw model of the neutrino mass sector. A similar mechanism can of course also lead to the EDM of the muon.

We find that two-loop diagrams give non-vanishing results for the $d_{e}$. However, EDMs are very much suppressed by the smallness of the neutrino mass as they first appear in $O\left(m_{e} m_{\nu}^{2} G_{F}^{2}\right)$ order, if the see-saw type relation between $m_{\nu}$, Dirac mass and heavy Majorana mass is imposed. Thus, for any phenomenologically motivated choice of $m_{\nu}$ the result for $d_{e}$ (and $d_{\mu}$ ) turns out to be much smaller than current or projected experimental sensitivity to EDMs. We also note that the fine-tuning in the neutrino mass sector may lead to up to ten orders of magnitude enhancement in $d_{e}$, which is still not enough to bring it within the experimental reach in the near future. 


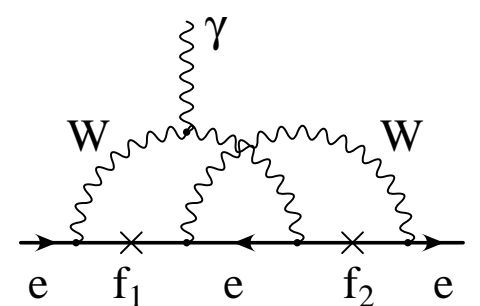

(1)

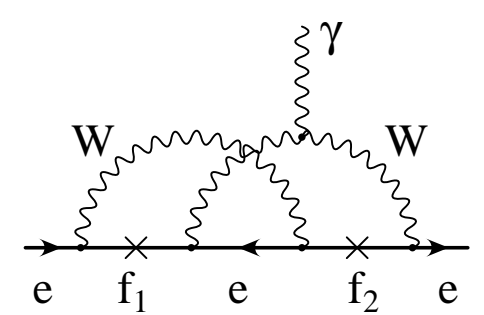

$(2)$

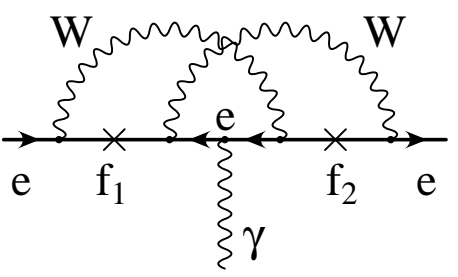

(3)

Figure 2: Contributions to the electron EDM in a model with Majorana masses of neutrinos. $f_{1,2}$ denote all possible neutrinos (see text). Crosses denote insertions of leptonnumber violating mass parameters. Note that the direction of the internal electron line is opposite to the external ones.

\section{Description of the model}

We take one standard model generation: $\left(\begin{array}{c}\nu_{L} \\ e_{L}\end{array}\right), e_{R}$, and two singlet heavy neutrinos $N_{1,2}$. The latter do not participate in electroweak interactions; in particular, the charged current sector is described by the Lagrangian

$$
\left.\mathcal{L}_{c c}=\frac{g}{\sqrt{2}}\left(\bar{\nu}_{L} W^{+} e_{L}+\text { (H.c. }\right)\right)
$$

The mass sector Lagrangian for fermions is

$$
\begin{aligned}
-\mathcal{L}_{M}= & m_{e}\left(\bar{e}_{L} e_{R}+\bar{e}_{R} e_{L}\right) \\
& +\frac{M_{1}}{2}\left(\bar{N}_{1}^{c} N_{1}+\bar{N}_{1} N_{1}^{c}\right)+\frac{M_{2}}{2}\left(\bar{N}_{2}^{c} N_{2}+\bar{N}_{2} N_{2}^{c}\right) \\
& +m_{1}\left(e^{i \phi_{1}} \bar{N}_{1} \nu_{L}+e^{-i \phi_{1}} \bar{\nu}_{L} N_{1}\right) \\
& +m_{2}\left(e^{i \phi_{2}} \bar{N}_{2} \nu_{L}+e^{-i \phi_{2}} \bar{\nu}_{L} N_{2}\right) .
\end{aligned}
$$

Here $\psi^{c} \equiv \gamma_{0} C \psi^{*} ; M_{1,2}$ and $m_{1,2}$ are defined in terms of real positive Yukawa couplings $y_{1,2}$ and the electroweak vacuum expectation value $v$,

$$
m_{1,2} \equiv \frac{y_{1,2} v}{\sqrt{2}}
$$

We use the freedom of phase choice for $\nu_{L}$ and $e_{R, L}$ to redefine

$$
\nu_{L} \rightarrow e^{-i \phi_{2}} \nu_{L}
$$

We see that there is only one physical CP violating phase $\eta \equiv \phi_{1}-\phi_{2}$.

Before we explore the physical manifestation of $\eta$, we determine the mass eigenstates of neutrinos. We will use them to compute the EDM of the electron. We use the identity

$$
\begin{aligned}
\bar{\nu}_{L} N & =\frac{1}{2}\left(\bar{\nu}_{L} N+\bar{N}^{c} \nu_{L}^{c}\right) \\
\bar{N} \nu_{L} & =\frac{1}{2}\left(\bar{N} \nu_{L}+\bar{\nu}_{L}^{c} N^{c}\right)
\end{aligned}
$$


to rewrite the neutrino mass matrix as

$$
\begin{aligned}
-\mathcal{L}_{M, \nu} & =\frac{1}{2}\left(\bar{\nu}_{L}, \bar{N}_{1}^{c}, \bar{N}_{2}^{c}\right) \mathcal{M}\left(\begin{array}{c}
\nu_{L}^{c} \\
N_{1} \\
N_{2}
\end{array}\right)+(\text { H.c. }) \\
\mathcal{M} & \equiv\left(\begin{array}{ccc}
0 & m_{1} e^{i \eta} & m_{2} \\
m_{1} e^{i \eta} & M_{1} & 0 \\
m_{2} & 0 & M_{2}
\end{array}\right)
\end{aligned}
$$

We use $M_{1,2} \gg m_{1,2}$ to approximately diagonalize the neutrino mass matrix. We define neutrino mass eigenstates: light $\nu$ and heavy $n_{1,2}$,

$$
\left(\begin{array}{c}
\nu_{L}^{c} \\
N_{1} \\
N_{2}
\end{array}\right)=V\left(\begin{array}{c}
\nu^{c} \\
n_{1} \\
n_{2}
\end{array}\right)
$$

where $V$ is the matrix diagonalizing $\mathcal{M}$. Approximately,

$$
\begin{aligned}
V & =\left(\begin{array}{ccc}
1 & \frac{m_{1} e^{i \eta}}{M_{1}} & \frac{m_{2}}{M_{2}} \\
-\frac{m_{1} e^{i \eta}}{M_{1}} & 1 & 0 \\
-\frac{m_{2}}{M_{2}} & 0 & 1
\end{array}\right) \\
V^{T} \mathcal{M} V & =\left(\begin{array}{ccc}
-\frac{m_{1}^{2} e^{2 i \eta}}{M_{1}}-\frac{m_{2}^{2}}{M_{2}} & o_{2} & o_{2} \\
O_{2} & M_{1}+o_{1} & o_{1} \\
o_{2} & o_{1} & M_{2}+o_{1}
\end{array}\right)
\end{aligned}
$$

where we denote small corrections by $o_{n} \equiv \mathcal{O}\left(m\left(\frac{m}{M}\right)^{n}\right)$. Two things are worth noting:

- Heavy neutrinos $n_{1,2}$ participate in charged current interactions described by eq. (1), through their presence in $\nu_{L}$, eq. (7):

$$
\nu_{L}^{c} \simeq \nu^{c}+\frac{m_{1} e^{i \eta}}{M_{1}} n_{1}+\frac{m_{2}}{M_{2}} n_{2}
$$

- The $\mathrm{CP}$ violating phase enters through the complex mass of $\nu$ and through the charged current interaction of $n_{1}$.

The Majorana mass of the light (active) neutrino is given by the following relation,

$$
m_{\nu}=\left|\frac{m_{1}^{2} e^{2 i \eta}}{M_{1}}+\frac{m_{2}^{2}}{M_{2}}\right| .
$$

Since experiments with light neutrinos give information about $m_{\nu}$, it is convenient to keep this parameter fixed, while allowing $m_{1,2}, M_{1,2}$ and $\eta$ to vary. In doing this, we would have to distinguish two possibilities.

- See-saw relation, when both contributions in (10) are on the order or smaller than $m_{\nu}$ 
- Cancellation of two terms in $m_{\nu}$ which we would term as a "fine-tuned case", although it could be a result of some symmetry that suppresses the determinant of the mass matrix (6). For this cancellation to happen, one has to have $m_{1}^{2} / M_{1} \simeq m_{2}^{2} / M_{2}$ and phase $\eta$ close to $\pi / 2$.

We would like to note that theoretically one could have a fine-tuned case even for $m_{1,2} \sim$ $M_{1,2}$. This, however, would also result in large admixtures of heavy $n_{1}$ and $n_{2}$ neutrinos in the original left-handed neutrino. In a more realistic model with three active neutrino species, such admixtures could lead to a non-universality of charged currents, modification of the invisible $Z$ decay width, etc. With the accuracy of electroweak data, we impose the bound on the size of the mixing angle:

$$
\frac{m_{1}}{M_{1}}, \frac{m_{2}}{M_{2}} \lesssim \mathcal{O}\left(\frac{1}{10}\right)
$$

\section{Computation of the electron EDM at two loops}

To define the electron EDM, consider the general matrix element of an electromagnetic current between spin 1/2 fermions,

$$
\begin{aligned}
\bar{u}_{f}(p) & \left\{F_{1}(t) \gamma_{\mu}-\frac{i}{2 m} F_{2}(t) \sigma_{\mu \nu} q^{\nu}+\frac{1}{m} F_{3}(t) q_{\mu}\right. \\
+ & \left.\gamma_{5}\left[G_{1}(t) \gamma_{\mu}-\frac{i}{2 m} G_{2}(t) \sigma_{\mu \nu} q^{\nu}+\frac{1}{m} G_{3}(t) q_{\mu}\right]\right\} u_{i}(p+q),
\end{aligned}
$$

with $t \equiv q^{2}$. The EDM $d_{e}$ of an electron of charge $e$ is given by

$$
d_{e}=-\frac{i e}{2 m_{e}} G_{2}(0)
$$

The most notable property of all diagrams in Figs. 1 and 2, potentially contributing to the CP-odd amplitude, is the effective antisymmetrization over the neutrino propagation, $f_{1}$ and $f_{2}$. This is because the CP-odd part of a diagram with the selection of eigenstates $f_{1}$ and $f_{2}$ is always opposite to the CP-odd part of the diagram with interchanged flavours. As a result of this antisymmetrization, in the expansion over small $q$ the first class of diagrams, Fig. 1a and 1b, turns out to be proportional to the cube of the photon momentum $O\left(q^{3}\right)$, while $G_{2}(0)$ vanishes identically. A more detailed explanation of the cancellation of EDMs can be found in Ref. [8, 13, 11].

Within the model we are considering, the non-vanishing diagrams contributing to $d_{e}$ are shown in Fig. 2. Their evaluation is simplified if we assume the most natural mass

hierarchy, $\frac{m_{1,2}^{2}}{M_{1,2}} \ll m_{e} \ll M_{W} \ll M_{1,2}$. We use the notation $M=\frac{M_{1}+M_{2}}{2}$ and $\Delta M=$ $M_{2}-M_{1}$ and assume $|\Delta M| \ll M$ for calculational convenience.

First, we treat the case when $f_{1,2}$ are both heavy (some details of derivations of these results are given in the Appendix),

$$
\Delta d_{e}(\text { heavy-heavy })=e\left(\frac{G_{F}}{16 \pi^{2}}\right)^{2} m_{e} \frac{\Delta M}{M} \frac{m_{1}^{2}}{M} \frac{m_{2}^{2}}{M} M^{-4 \epsilon}\left(\frac{16}{3 \epsilon}-\frac{364}{9}+\frac{112}{27} \pi^{2}\right) \sin 2 \eta .
$$


Next, we obtain the contribution when one of $f_{i}$ is the light state $\nu$,

$$
\left.\Delta d_{e} \text { (heavy-light }\right)=e\left(\frac{G_{F}}{16 \pi^{2}}\right)^{2} m_{e} \frac{\Delta M}{M} \frac{m_{1}^{2}}{M} \frac{m_{2}^{2}}{M} M^{-6 \epsilon} M_{W}^{2 \epsilon}\left(-\frac{16}{3 \epsilon}+\frac{104}{9}\right) \sin 2 \eta \text {. }
$$

The sum of both contributions is finite,

$$
\begin{aligned}
d_{e} & =\Delta d_{e} \text { (heavy-heavy) }+\Delta d_{e} \text { (heavy-light) } \\
& =e\left(\frac{G_{F}}{16 \pi^{2}}\right)^{2} m_{e} \frac{\Delta M}{M} \frac{m_{1}^{2}}{M} \frac{m_{2}^{2}}{M}\left(\frac{32}{3} \ln \frac{M}{M_{W}}-\frac{260}{9}+\frac{112}{27} \pi^{2}\right) \sin 2 \eta
\end{aligned}
$$

Our answer shows that the electron EDM first appears at $O\left(m_{e} m_{1}^{2} m_{2}^{2} M^{-2} G_{F}^{2}\right)$ level, which is essentially the same as $O\left(m_{e} m_{\nu}^{2} G_{F}^{2}\right)$ if the see-saw relations $m_{1,2}^{2} / M \sim O\left(m_{\nu}\right)$ are in place.

In a slightly different model, with two generations of active neutrinos with masses $m_{\nu 1}$ and $m_{\nu 2}$ there is an additional possibility of $f_{1}$ and $f_{2}$ both being light. It is easy to see, however, that in the case of (light-light) diagram the contribution to $d_{e}$ is parametrically much smaller than Eq. (16), being suppressed by four powers of light neutrino mass $m_{\nu 1} m_{\nu 2}\left(m_{\nu 1}^{2}-m_{\nu 2}^{2}\right)$. Thus, the generalization to the case of three generations would not bring any parametric change to the size of $d_{e}$. Fixing $m_{\nu}$, we can also allow $m_{1,2}$ and $M_{1,2}$ to vary. If $M_{1,2}$ becomes smaller than the electroweak scale, $d_{e}$ acquires additional suppression by $M^{2} / M_{W}^{2}$. This proves that (16) with the chosen hierarchy of scales represents the largest possible contribution to $d_{e}$ at fixed $m_{\nu}$.

\section{Discussion and Conclusions}

What is the largest numerical value of the answer (16)? For the see-saw type relation, in the absence of substantial cancellations between the two terms in (10), we can use the following inequality

$$
\left|\frac{m_{1}^{2}}{M} \frac{m_{2}^{2}}{M} \sin 2 \eta\right| \lesssim m_{\nu}^{2}
$$

This leads to an extremely tight theoretical bound on the possible size of the EDM,

$$
\left|d_{e}\right| \lesssim e\left(\frac{G_{F}}{16 \pi^{2}}\right)^{2} m_{e} m_{\nu}^{2} \frac{|\Delta M|}{M}\left(10.7 \times \ln \frac{M}{M_{W}}+12.1\right), \quad \text { see-saw case. }
$$

Using $|\Delta M| \sim M \sim 10^{16} \mathrm{GeV}$, and the Particle Data Group book [21] bound on the mass of the electron neutrino, $m_{\nu}<3 \mathrm{eV}$, we arrive at our final numerical result for the see-saw case,

$$
\left|d_{e}\right|<1.5 \times 10^{-43} \text { e cm, see-saw case. }
$$

Not only is this number much smaller than the most optimistic accuracy of future electron EDM searches [22], but also it is significantly smaller than EDMs induced by the KM phase from the quark sector. 
The fine-tuned case deserves special consideration, as the numerical answer for $d_{e}$ can be significantly larger than (19). Introducing another angle $\delta=\pi / 2-\eta$, which has to be small for the fine-tuning of $m_{\nu}$ to happen, we rewrite the light neutrino mass squared as

$$
m_{\nu}^{2}=\frac{1}{M^{2}}\left(\left(m_{1}^{2}-m_{2}^{2}\right)^{2}+4 m_{1}^{2} m_{2}^{2} \delta^{2}\right)
$$

On the other hand, the answer for EDM contains a factor $\frac{m_{1}^{2} m_{2}^{2}}{M^{2}} \sin (2 \eta)=2 \frac{m_{1}^{2} m_{2}^{2}}{M^{2}} \delta$, which in view of Eq. (20) can be limited as

$$
\left|\frac{m_{1}^{2} m_{2}^{2}}{M^{2}} 2 \delta\right|<\frac{m_{1} m_{2}}{M} m_{\nu} \lesssim(0.1 \times 500 \mathrm{GeV}) m_{\nu}
$$

In this expression, we have used (11) and the upper bound on $m_{1,2}$ taken to be $\sim 500$ $\mathrm{GeV}$. The latter follows from the condition that Yukawa sector remains perturbative, i.e. $y_{1,2} \lesssim O(1-10)$. Comparing (17) and (21), we observe that the fine-tuned case may lead to up to $10^{10}$ enhancement relative to the see-saw case and the electron EDM may reach a value of

$$
d_{e} \lesssim 10^{-33} \text { e cm, fine-tuned case. }
$$

This value is still far from the existing or projected experimental accuracy but is much larger than the electron EDM induced by the KM phase.

In some models the effects of the CP-odd electron-nucleon interaction, $C_{S} \bar{N} N \bar{e} i \gamma_{5} e$ may dominate over the electron EDM contribution [23] in the atomic (molecular) EDM. An analysis of possible two-loop diagrams for $C_{S}$ shows that in the model with CP violation coming from Majorana neutrinos this is not the case, and the contribution of $C_{S}(\eta)$ to atomic EDM is smaller than (19) for the see-saw case and (22) for the fine-tuned case.

To summarize, we have calculated the contributions of the two-loop diagrams to the EDM of the electron in non-supersymmetric models with $\mathrm{CP}$ violation in the lepton sector and with Majorana masses for neutrinos. We notice that the non-zero result for $d_{e}$ can be obtained in a rather minimalistic way: with one generation of SM leptons and two righthanded neutrinos. In terms of the right-handed mass $M$ and Dirac mass $m_{D}$, the non-zero result appears in order $O\left(m_{e} m_{D}^{4} M^{-2} G_{F}^{2}\right)$, which is the largest possible result given the symmetries of the model. If the smallness of the light neutrino mass is achieved via usual see-saw relation without any fine-tuning, this answer is equivalent to $O\left(m_{e} m_{\nu}^{2} G_{F}^{2}\right)$ and numerically is extremely small. Numerical smallness of this result stems from the smallness of $m_{\nu}$, and the parametric suppression of EDM is very similar to the suppression of e.g. the SM amplitude for $\mu \rightarrow e \gamma$ decay. In the fine-tuned case, when the smallness of the light neutrino mass is achieved via near perfect cancellation of two contributions, the result for $d_{e}$ may become larger by many orders of magnitude, but still smaller than about $10^{-33} \mathrm{ecm}$, and therefore much smaller than the sensitivity of any EDM experiment in the foreseeable future.

We conclude that the KM-type models with Majorana neutrino masses do not have any impact on the EDM searches. Therefore, the only options of searching for CP-violation in the neutrino sector suggested by the leptogenesis scenario are the CP asymmetries in neutrino oscillations and not EDMs. Conversely, possible positive results from the future electron EDM searches could be an indication of leptogenesis combined with the soft supersymmetry breaking. 


\section{Acknowledgement}

This research was supported by the Science and Engineering Research Canada, and by the Collaborative Linkage Grant PST.CLG.977761 from the NATO Science Programme.

\section{A Details of the calculation of two-loop diagrams}

Here we give intermediate results for the sum of the three diagrams in Fig. 2, before we add and simplify contributions from $n_{1,2}$.

For the heavy-heavy case we get

$$
\begin{aligned}
\Delta d_{e}(\text { heavy-heavy })= & -\frac{i e^{5}}{2 m_{e} s_{W}^{4}} \frac{1}{\left(16 \pi^{2}\right)^{2}}\left(\frac{m_{1} m_{2}}{M_{1} M_{2}}\right)^{2}\left(e^{2 i \eta}-\text { complex conjugate }\right) \\
& \cdot\left(M_{2}-M_{1}\right) \frac{M m_{e}^{2}}{M_{W}^{4}} M^{-4 \epsilon}\left(\frac{1}{6 \epsilon}-\frac{91}{72}+\frac{7}{54} \pi^{2}\right) \\
\simeq & \left(\frac{G_{F}}{16 \pi^{2}}\right)^{2} m_{e} e \frac{\Delta M m_{1}^{2} m_{2}^{2}}{M^{3}} M^{-4 \epsilon}\left(\frac{16}{3 \epsilon}-\frac{364}{9}+\frac{112}{27} \pi^{2}\right) \sin 2 \eta .
\end{aligned}
$$

For the heavy-light case we find

$$
\begin{aligned}
&\left.\Delta d_{e} \text { (heavy-light }\right)=-\frac{i e^{5}}{2 m_{e} s_{W}^{4}} \frac{1}{\left(16 \pi^{2}\right)^{2}} \frac{m_{e}^{2}}{M_{W}^{4}} \\
& \cdot\left[\frac{m_{1}^{2}}{M_{1}^{2}} e^{2 i \eta} f\left(-\frac{m_{2}^{2}}{M_{2}}, M_{1}\right)+\frac{m_{2}^{2}}{M_{2}^{2}} f\left(-\frac{m_{1}^{2}}{M_{1}} e^{-2 i \eta}, M_{2}\right)-\text { complex conjugate }\right],
\end{aligned}
$$

where the function $f$ arises from the evaluation of the diagrams without coupling constants,

$f\left(m_{\nu}, M_{i}\right) \equiv m_{\nu} M_{i} M_{W}^{-4 \epsilon}\left(-\frac{1}{12 \epsilon} \ln \frac{M_{i}^{2}}{M_{W}^{2}}-\frac{5}{16 \epsilon}+\frac{13}{72} \ln \frac{M_{i}^{2}}{M_{W}^{2}}+\frac{1}{8} \ln ^{2} \frac{M_{i}^{2}}{M_{W}^{2}}-\frac{31}{48}+\frac{\pi^{2}}{36}\right)$.

Here $m_{\nu}$ and $M_{i}$ denote a light and a heavy neutrino mass insertion. Working to first order in the heavy neutrino mass splitting $\Delta M$, we get

$$
\begin{aligned}
\left.\Delta d_{e} \text { (heavy-light }\right)= & -\frac{i e^{5}}{2 m_{e} s_{W}^{4}} \frac{1}{\left(16 \pi^{2}\right)^{2}} \frac{m_{e}^{2}}{M_{W}^{4}} \\
& \cdot \frac{m_{1}^{2} m_{2}^{2}}{M^{3}} i \frac{\Delta M}{16} M_{W}^{-4 \epsilon}\left(-\frac{16}{3 \epsilon}+\frac{104}{9}+16 \ln \frac{M^{2}}{M_{W}^{2}}\right) \sin 2 \eta \\
= & \left(\frac{G_{F}}{16 \pi^{2}}\right)^{2} m_{e} e \frac{\Delta M m_{1}^{2} m_{2}^{2}}{M^{3}} M_{W}^{-4 \epsilon}\left(-\frac{16}{3 \epsilon}+\frac{104}{9}+16 \ln \frac{M^{2}}{M_{W}^{2}}\right) \sin 2 \eta
\end{aligned}
$$

We see that in the sum of contributions in (23) and (26), the divergences cancel and we obtain the final result, eq. (16). 


\section{B Asymptotic Expansions}

In this appendix, we show how the method of asymptotic expansions [24] is used to calculate the contributing diagrams for the electric dipole moment.

Two-loop Feynman diagrams containing more than one mass scale are difficult to solve analytically. Analytic expansions in small parameters, such as the ratio of lepton and weak boson masses, are used to reduce the calculations to integrals involving only one mass scale. The results are integrals which have analytic solutions. As an example of this technique, we use Feynman diagrams from Fig. 2 containing one light neutrino, $f_{1}$, of mass $m_{\nu}$ and one heavy neutrino, $f_{2}$, of mass $M$.

The momentum assignments for the calculation are illustrated in Fig. 3. Conservation of momentum at each vertex requires $p_{1}=p+k_{1}, p_{2}=p+k_{1}+k_{2}$ and $p_{3}=p+k_{2}$.

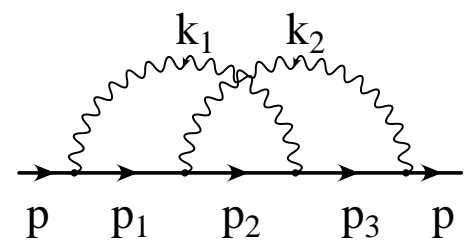

Figure 3: Momentum assignment for the EDM calculation.

In order to perform the expansions, all mass scales must be identified. For our calculation, the mass scales involved are $M \gg M_{W} \gg m_{e} \gg m_{\nu}$.

Once the mass scales are determined, the integral volumes are divided into regions so that the momentum flow through the internal lines are on the order of one of the mass scales. With one light and one heavy neutrino, there are four scenarios to be considered:

1. $k_{1}, k_{2} \ll M, M_{W}$

2. $k_{2} \sim M, k_{1} \ll M, M_{W}$

3. $k_{1}, k_{2} \sim M$

4. $k_{1}, k_{2} \sim M, k_{1}+k_{2} \sim m_{e}$

Within each region, the appropriate propagators can be expanded in a Taylor series to reduce the number of mass scales in the integrals.

\section{B.1 $k_{1}, k_{2} \ll M, M_{W}$}

In this momentum region, the heavy neutrino and the $W$ boson propagators can be expanded in a Taylor series. For example, the heavy neutrino propagator can be written as

$$
\frac{1}{k^{2}+M^{2}}=\frac{1}{M^{2}} \sum_{n=0}^{\infty}\left(\frac{k^{2}}{M^{2}}\right)^{n}
$$


since $k \ll M$.

The Taylor expansion reduces the diagram in Fig. 3 to that of Fig. 4. The two-loop diagram is reduced to the product of two one-loop diagrams. Each one-loop diagram has one mass scale and has an analytic solution.

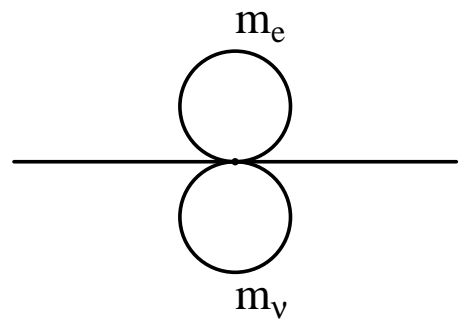

Figure 4: Reduction of a two-loop diagram to two one-loop diagrams for $k_{1}, k_{2} \ll M, M_{W}$. The solid lines represent massive propagators.

\section{B.2 $k_{2} \sim M, k_{1} \ll M, M_{W}$}

When one momentum is large and the other is small, the propagators are expanded in all momenta except for the large momentum. This reduces the calculation to the diagrams in Fig. 5, a product of two one-loop diagrams. Clearly, the first diagram has only one mass scale involved. In the second diagram, partial fractions are used to further separate the mass scales. In particular, we use the identity

$$
\frac{1}{k^{2}+M^{2}} \frac{1}{k^{2}+M_{W}^{2}}=\frac{1}{M_{W}^{2}-M^{2}}\left(\frac{1}{k^{2}+M^{2}}-\frac{1}{k^{2}+M_{W}^{2}}\right)
$$

The resulting diagrams can be computed analytically.

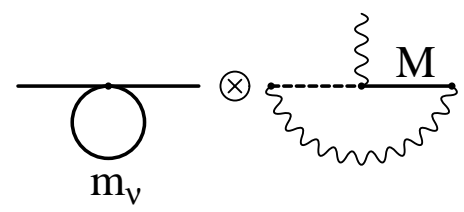

Figure 5: Reduction of a two-loop diagram to two one-loop diagrams for $k_{2} \sim M, k_{1} \ll$ $M, M_{W}$. The dashed line represents a massless propagator.

\section{B.3 $k_{1}, k_{2} \sim M$}

If both momenta are large, a reduction is made to the form of Fig. 6. Notice however, that there are still two mass scales in the diagram. Thus, we use the assumption that 
$M \gg M_{W}$ for further expansion to reduce the diagram down to a one mass scale diagram.

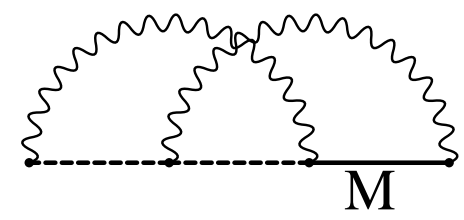

Figure 6: Taylor Expansion for $k_{1}, k_{2} \sim M$.

\section{B.4 $k_{1}, k_{2} \sim M, k_{1}+k_{2} \sim m_{e}$}

The last case reduces to Fig. 7 but does not contribute to the $d_{e}$ in the leading order.

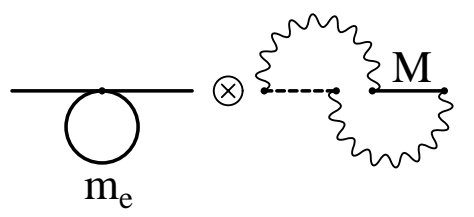

Figure 7: Taylor expansion for the case $k_{1}+k_{2} \ll M$.

After integration over the initial ranges is performed, the contributions from all momentum regions are summed to produce eq. (15).

Using a similar analysis, eq. (14) was computed for the diagrams with two heavy neutrinos.

\section{References}

[1] B. Aubert et al. [BABAR Collaboration], Phys. Rev. Lett. 87 (2001) 091801.

[2] K. Abe et al. [Belle Collaboration], Phys. Rev. Lett. 87 (2001) 091802.

[3] J. H. Christenson, J. W. Cronin, V. L. Fitch and R. Turlay, Phys. Rev. Lett. 13 (1964) 138.

[4] M. Kobayashi and T. Maskawa, Prog. Theor. Phys. 49 (1973) 652.

[5] P. G. Harris et al., Phys. Rev. Lett. 82 (1999) 904.

[6] B. C. Regan et al., Phys. Rev. Lett. 88 (2002) 07180.

[7] M. V. Romalis, W. C. Griffith and E. N. Fortson, Phys. Rev. Lett. 86 (2001) 2505. 
[8] E. P. Shabalin, Sov. J. Nucl. Phys. 28 (1978) 75 [Yad. Fiz. 28 (1978) 151].

[9] I. B. Khriplovich, Phys. Lett. B 173 (1986) 193.

[10] A. Czarnecki and B. Krause, Phys. Rev. Lett. 78 (1997) 4339.

[11] M. E. Pospelov, Phys. Lett. B 328 (1994) 441.

[12] M. B. Gavela et al., Phys. Lett. B 109 (1982) 215;

I. B. Khriplovich and A. R. Zhitnitsky, Phys. Lett. B 109 (1982) 490.

[13] I. B. Khriplovich and M. E. Pospelov, Sov. J. Nucl. Phys. 53 (1991) 638.

[14] M. Fukugita and T. Yanagida, Phys. Lett. B 174 (1986) 45.

[15] V. A. Kuzmin, V. A. Rubakov and M. E. Shaposhnikov, Phys. Lett. B 155 (1985) 36.

[16] J. N. Bahcall, M. C. Gonzalez-Garcia and C. Pena-Garay, JHEP 0302 (2003) 009.

[17] D. Ng and J. N. Ng, Mod. Phys. Lett. A 11 (1996) 211.

[18] A. Pilaftsis, Int. J. Mod. Phys. A 14 (1999) 1811.

[19] S. Dimopoulos and L. J. Hall, Phys. Lett. B 344 (1995) 185;

P. Ciafaloni, A. Romanino and A. Strumia, Nucl. Phys. B 458 (1996) 3;

K. S. Babu, B. Dutta and R. N. Mohapatra, Phys. Rev. Lett. 85 (2000) 5064;

J. R. Ellis, J. Hisano, M. Raidal and Y. Shimizu, Phys. Lett. B 528 (2002) 86;

B. Dutta and R. N. Mohapatra, Phys. Rev. D 68 (2003) 113008;

A. Bartl, W. Majerotto, W. Porod and D. Wyler, Phys. Rev. D 68 (2003) 053005;

Y. Farzan and M. E. Peskin, arXiv:hep-ph/0405214;

See also Y. K. Semertzidis, arXiv:hep-ex/0401016.

[20] A. de Gouvea, B. Kayser and R. N. Mohapatra, Phys. Rev. D 67 (2003) 053004.

[21] K. Hagiwara et al. [Particle Data Group Collaboration], Phys. Rev. D 66 (2002) 010001.

[22] J. J. Hudson, B. E. Sauer, M. R. Tarbutt and E. A. Hinds, Phys. Rev. Lett. 89 (2002) 023003;

D. Kawall, F. Bay, S. Bickman, Y. Jiang and D. DeMille, Phys. Rev. Lett. 92 (2004) 133007;

S. K. Lamoreaux, arXiv:nucl-ex/0109014.

[23] S. M. Barr, Phys. Rev. Lett. 68 (1992) 1822;

O. Lebedev and M. Pospelov, Phys. Rev. Lett. 89 (2002) 101801.

[24] For reviews and further references see, for example,

F. V. Tkachov, Sov. J. Part. Nucl. 25, 649 (1994) [arXiv:hep-ph/9701272];

V. A. Smirnov, Applied asymptotic expansions in momenta and masses, Springer Verlag (Berlin), 2002;

K. G. Chetyrkin, preprint MPI-Ph/PTh 13/91 (unpublished);

G. B. Pivovarov and F. V. Tkachov, Int. J. Mod. Phys. A8 (1993) 2241 [arXiv:hep$\mathrm{ph} / 9612287]$. 